Published May 27

OPEN
ACCESS

\title{
Intestinal microbial diversity and functional analysis of Urechis unicinctus from two different habitats: pond polycultured with Penaeus japonicus and coastal zone
}

\author{
Yongzheng Tang ${ }^{1}$, Shuai $\mathrm{Ma}^{1}$, Yihao Liu ${ }^{2}$, Yongrui $\mathrm{Pi}^{1, *}{ }^{1,}$ Ying Liu ${ }^{1}$, Ye Zhao ${ }^{1}$ \\ ${ }^{1}$ School of Ocean, Yantai University, Yantai 264005, PR China \\ ${ }^{2}$ Shandong Key Laboratory of Marine Ecological Restoration, Shandong Marine Source and Environment Research Institute, \\ Yantai 264006, PR China
}

\begin{abstract}
Urechis unicinctus is an important commercial and ecological invertebrate that has potential applications in the study of marine invertebrate evolution and marine pharmaceutical development. Here we analyzed the intestinal microbial diversity of $U$. unicinctus from 2 different habitats using 16S rDNA 454 high-throughput sequencing. The dominant phyla were Proteobacteria, Bacterioidetes, Firmicutes, and Actinobacteria in gut samples of U. unicinctus, which significantly differed from those in its 2 habitats (i.e. intertidal mudflat and pond polyculture). Exceptions were Proteobacteria, Firmicutes and Bacterioidetes, which were the dominant phyla in the sediment and water samples. The top 15 genera in the gut samples did not show any significant differences between the 2 habitats. Functional analysis of the intestinal microbial community showed that metabolism, including carbohydrate and amino acid metabolism, was the most important function. Methane metabolism was one of the main components of energy metabolism. The gut microbes also played an important role in environmental and genetic information processing, cellular processes, etc. These findings provide an understanding of gut microbiome composition and diversity in $U$. unicinctus from 2 different habitats. Our results can inform the management of animal health in $U$. unicinctus farming activities and further gut microecology research.
\end{abstract}

KEY WORDS: Urechis unicinctus · Intestinal microbes $\cdot$ Community diversity $\cdot$ Functional analysis

\section{INTRODUCTION}

Aquaculture is one of the most rapidly growing traditional agricultural industries, providing approximately half of the fish consumed by humans (Lu et al. 2020). The requirement for aquatic products has supported the fast expansion of aquaculture, which has also led to huge economic benefits (Zhao et al. 2020). Among aquacultured resources, Urechis unicinctus is one of the most valuable products in Asia due to its high nutritional and medicinal values. $U$. unicinctus

${ }^{*}$ Corresponding author: yrpi@outlook.com has the capacity to tolerate and utilize sulfide, a toxicant (Shi et al. 2012); this capacity has ecological value for improving the biological suitability of sulfur-containing substrates in coastal substrates. Polyculture systems can improve biological diversity, enhance the utilization of input material, strengthen the self-purifying ability of aquaculture water, improve economic efficiency, and reduce environmental pollution. U. unicinctus are always polycultured with Penaeus japonicus or Stichopus japonicus (Yuan et al. 2015), and can make full use of a large amount of

(1) The authors 2021. Open Access under Creative Commons by Attribution Licence. Use, distribution and reproduction are unrestricted. Authors and original publication must be credited. 
nutrients and organics, reduce the content of sulfide, and improve the breeding environment significantly.

The gut microbiome is an ecological community of commensal, symbiotic, and pathogenic microorganisms inhabiting the gastrointestinal tract, including bacteria, viruses, parasites, fungi, archaea, and protists (Tremaroli \& Bäckhed 2012, Wang et al. 2016). It is estimated that approximately $10^{14}$ bacterial organisms comprising 500 1000 unique species form a synergistic relationship with the host (Ley et al. 2005). Intestinal microorganisms can not only assist the host in digestion and improve the utilization efficiency of nutrients, but they can also help the host to remove toxins and maintain health, control the colonization of intestinal pathogens and parasites, regulate endocrine function, and improve immune function (Tarnecki et al. 2017, Zhao et al. 2019, Zheng et al. 2019, Patil et al. 2020). Therefore, intestinal microorganisms and their interactions with the host have become research 'hotspots' in the fields of microbiology and medicine.

Intestinal microbes have some unique metabolic pathways, and they can establish a complex metabolic network with the host through special interactions (Winter \& Bäumler 2014). However, our current understanding of intestinal microbes is still very limited, and only $20 \%$ of the genes in the human intestinal metagenome have been annotated with specific functions (Huttenhower et al. 2012). Therefore, intestinal microbes are an important resource pool for studying new metabolic pathways and exploring new enzyme resources. Compared with terrestrial organisms, very little research has been conducted on the intestinal microbial diversity and function of marine organisms, and previous studies have mainly focused on fish (Mouchet et al. 2012, Zheng et al. 2018, Parris et al. 2019, Wilkes Walburn et al. 2019, Zheng et al. 2019) and sea cucumbers (Song et al. 2019, Yang et al. 2019, Zhao et al. 2019, Liu et al. 2020).

U. unicinctus (Echiuroidea, Echiurida, Urchidae, Urechis), known as the fat innkeeper worm or Chinese penis fish, is a coastal benthic organism that inhabits U-shaped burrows in intertidal and subtidal mudflats in Russia, Japan, the Korean Peninsula, and China (Ma et al. 2016; Bi et al. 2013). Adult Urechis are active filter feeders in that they form a finemeshed mucus bag through which they draw water by peristaltic contractions to collect food particles (Jumars et al. 2015, Wang et al. 2018). No reports have investigated the intestinal microbial diversity of $U$. unicinctus or conducted a functional analysis of the microbiota, despite its potentially high research and application value.
Intestinal microbiota are essential for host health and greatly influence the growth and development of the host by affecting the performance of the host, including its physiological, immunological, nutritional, and metabolic status (Tarnecki et al. 2017, Zhao et al. 2019, Zheng et al. 2019, Patil et al. 2020). Intestinal microbes have some unique metabolic pathways, and they can establish complex metabolic networks with the host; it is therefore of great significance to clarify the microbial diversity and functions in the intestinal environment of $U$. unicinctus. For this study, we selected $U$. unicinctus from 2 different habitats: (1) in pond polyculture with $P$. japonicus and (2) a coastal intertidal flat to investigate intestinal microbial diversity and conduct a functional analysis by $16 \mathrm{~S}$ rDNA 454 high-throughput sequencing technique. The goal of this research was to (1) assess the difference in bacterial communities between the 2 different $U$. unicinctus habitats and screen out discriminatory taxa, (2) analyze intestinal microbial function, and (3) evaluate the correlation between gut microbiota and the bacterial communities in the 2 habitats.

\section{MATERIALS AND METHODS}

\subsection{Animals and sampling}

Urechis unicinctus with a mean fresh mass of $29.0 \pm$ 4.4(SD) $g$ were collected from a coastal intertidal flat around Furong Island, Laizhou Bay, Yantai, China. The worms occupied burrows below the low-tide line, at a depth of $4 \mathrm{~m}$. Living worms were maintained in containers with clean seawater for approximately $2 \mathrm{~h}$ before being transported to the laboratory. Upon arrival at the lab, the worms were euthanized and dissected under sterile conditions, and the intestinal contents were collected in sterilized storage tubes and stored at $-80^{\circ} \mathrm{C}$ for later use. Water and sediment samples were collected from the surrounding environment, frozen immediately in a liquid nitrogen tank, and then stored at $-80^{\circ} \mathrm{C}$ for later use. The worms, water, and sediment samples from the coastal flat are labeled SUU, SW, and SS, respectively.

$U$. unicinctus with a mean fresh mass of $16.6 \pm 5.0$ (SD) $g$ were collected from a seawater aquaculture pond polycultured with Penaeus japonicus, at a depth of 1.0-1.5 m. The pretreatment procedures were the same as described above. The worms, water, and sediment samples from the pond polyculture are labeled PUU, PW, and PS, respectively. 


\subsection{DNA extraction and PCR amplification}

We used an E.Z.N.A.® soil DNA Kit (Omega Biotek) to extract the total DNA of all the samples, following the manufacturer's protocols. A NanoDrop 2000 UV-vis spectrophotometer (Thermo Scientific) was employed to determine the concentration and purification of final DNA, and $1 \%$ agarose gel electrophoresis was applied to check the DNA quality. Using a thermocycler PCR system (GeneAmp 9700), the primers 338F and 806R (Chen et al. 2020) were used to amplify the V3-V4 hypervariable regions of the $16 \mathrm{~S}$ rRNA gene of all samples. The PCR reactions were conducted according to the literature (Zhao et al. 2019, Xu et al. 2020), and the PCR products were excised from a $2 \%$ agarose gel. An AxyPrep DNA Gel Extraction Kit (Axygen Biosciences) was used for further purification, and DNA was quantified using Quanti-Fluor ${ }^{\mathrm{TM}}-\mathrm{ST}$ (Promega).

\subsection{Sequencing on the Illumina MiSeq platform}

The purified amplicons were submitted to the Majorbio Bio-Pharm Technology Co. Ltd. (Shanghai, China) following standard protocols. They were then pooled in equimolar amounts and paired-end sequenced $(2 \times 300)$ on an Illumina MiSeq platform (Zhao et al. 2019, Xu et al. 2020).

\subsection{Bioinformatic data analysis}

Raw fastq files were quality-filtered by Trimmomatic and merged by FLASH using UPARSE (Zhao et al. 2019, Xu et al. 2020, Kong et al. 2021). Based on the Silva (SSU123) 16S rRNA database, each 16S rRNA gene sequence was analyzed by the RDP Classifier algorithm (http:// rdp.cme.msu.edu/), with a confidence threshold of $70 \%$. Richness and diversity, the community structure, and functional analysis were performed using the free online Majorbio Cloud Platform (www.majorbio.com).

KEGG orthology (KO), cluster of orthologous groups (COG), and pathways of the samples from amplicon sequencing results were predicted using the Phylogenetic Investigation of Communities by Reconstruction of Unobserved States (PICRUSt) based on the Greengenes 13.5 database (Langille et al. 2013). All software packages were freely available on the Majorbio Cloud Platform.

\subsection{Data analysis}

The relationship between the selected taxonomical groups (abundant phyla, genera, classes, orders, or families), the observed operational taxonomic units (OTUs), and the bacterial community index were calculated using SPSS 18.0 software. All statistical analyses were conducted using SPSS software (version 18.0). A value of $\alpha=0.05$ was accepted as the criterion for statistical significance. The homogeneity of variance of all data was tested by Levene's test and the normality of residuals was tested by ShapiroWilk test. One-way ANOVA was employed to conduct multiple-group comparisons, and Student's $t$-test (2-tailed test) was used to conduct 2-group comparisons. Data are presented as means $\pm \mathrm{SD}$.

\section{RESULTS}

The raw reads were deposited into the NCBI Sequence Read Archive (SRA) database under accession number PRJNA681016. A total of 902871 optimized sequences were obtained, from 2 intestinal samples, 2 water samples, and 2 sediment samples (1 sample each from the coastal zone and the polyculture pond, with 3 replicates for each sample) (Table 1). The numbers of clean sequences in the 2 different habitats and Urechis unicinctus gut were significantly different; the number of sequences in the pond samples was much lower than that from the coastal samples. After rarefaction to equal sequencing depth, a total of 4945 OTUs were obtained from all samples. The OTU numbers in SUU, PUU, SS, PS, SW, and PW were $513 \pm 77,618 \pm 258,1738 \pm 68$, $1783 \pm 79,1074 \pm 168$, and $445 \pm 21$, respectively. The OTU numbers in the sediment samples were larger than in the water samples and the gut of $U$. unicinctus. We identified 52 OTUs that were shared among all samples, and only a few unique OTUs (32) were found in PW samples, indicating that relatively few microorganisms inhabited PW. Intestine samples of $U$. unicinctus living in the 2 habitats had only 66 OTUs in common, suggesting significant differences in the gut bacteria between the 2 habitats (see Figs. S1 \& S2 in the Supplement at www.int-res.com/ articles/suppl/q013p211_supp.pdf).

\subsection{Richness and diversity analysis}

The richness and diversity of the microbial community can be studied through alpha diversity analysis, 
Table 1. Richness, diversity, and coverage estimates obtained at genetic distances of $3 \%$. Data are presented as means \pm SD. SUU (PUU): gut samples of Urechis unicinctus collected from the coast (collected from a pond polyculture); SS (PS): sediment samples collected from the coast (pond polyculture); SW (PW): water samples collected from the coast (pond polyculture);

OTUs: operational taxonomic units; ACE: abundance-based coverage estimators

\begin{tabular}{|lccccccc|}
\hline $\begin{array}{l}\text { Sample } \\
\text { ID }\end{array}$ & $\begin{array}{c}\text { Effective } \\
\text { sequences }\end{array}$ & OTUs & ACE & Chao & Shannon & $\begin{array}{c}\text { Simpson } \\
\text { coverage }\end{array}$ \\
\hline SUU & $56063 \pm 2473$ & $513 \pm 77$ & $521 \pm 75$ & $526 \pm 70$ & $4.43 \pm 0.22$ & $0.052 \pm 0.013$ & 0.9997 \\
PUU & $52192 \pm 4670$ & $618 \pm 258$ & $628 \pm 259$ & $643 \pm 261$ & $4.85 \pm 0.27$ & $0.036 \pm 0.006$ & 0.9995 \\
SS & $47977 \pm 3739$ & $1738 \pm 68$ & $2190 \pm 56$ & $2138 \pm 57$ & $4.46 \pm 0.06$ & $0.087 \pm 0.006$ & $0.983 \pm 0.006$ \\
PS & $44515 \pm 2865$ & $1783 \pm 79$ & $2225 \pm 83$ & $2204 \pm 60$ & $4.42 \pm 0.15$ & $0.085 \pm 0.014$ & $0.986 \pm 0.001$ \\
SW & $52367 \pm 3622$ & $1074 \pm 168$ & $1969 \pm 208$ & $1655 \pm 143$ & $4.44 \pm 0.10$ & $0.039 \pm 0.016$ & $0.990 \pm 0.003$ \\
PW & $47843 \pm 2161$ & $445 \pm 21$ & $877 \pm 106$ & $676 \pm 54$ & $3.44 \pm 0.02$ & $0.066 \pm 0.001$ & 0.995 \\
\hline
\end{tabular}

including a series of indices used to estimate species abundance and diversity of the environmental community (Table 1). Abundance-based coverage estimators (ACE) and Chao indices indicate the community richness of the samples (see Text S1 in the Supplement for details of how these indices were calculated). In both habitats, species richness was highest in the sediment samples. The gut bacterial community richness of $U$. unicinctus living in the pond was higher than that of $U$. unicinctus inhabiting the mudflat. The bacterial community richness of SW was much higher than that of PW; the ACE and Chao values of the SW samples were $1969 \pm 208$ and 1655 \pm 143 , nearly 2.5 times those of the PW samples. Shannon and Simpson indices reflect community diversity. Except for the PW, the Shannon and Simp- son indices showed no significant differences between the gut and habitat samples. Good's coverage index of all samples was $>0.98$, indicating that the OTUs of each sample were well captured.

\subsection{Bacterial composition of the intestine and the habitat}

We identified 58 different bacterial phyla from all samples. The dominant bacterial phyla that appeared in all samples were Proteobacteria, Bacteroidetes, Firmicutes, Actinobacteria, Cyanobacteria, Chloroflexi, and Acidobacteria (Figs. 1a \& S3). Among all samples, the abundance of Proteobacteria in SUU was the highest, and the abundance of Bacteroidetes was


Fig. 1. Comparison of the relative abundances of major bacterial phyla in samples collected from the intestine and habitats of Urechis unicinctus. (a) Relative abundance of the top 15 phylum-level bacterial communities in all samples. (b) Top 10 phyla shared between the intestine and habitat samples. Data are presented as means $\pm \mathrm{SD}$. One-way ANOVA was used to detect significant differences $\left({ }^{*} 0.01<\mathrm{p} \leq 0.05 ;{ }^{* *} 0.001<\mathrm{p} \leq 0.01 ;{ }^{* * *} \mathrm{p} \leq 0.001\right)$. Sample group abbreviations as in Table 1 


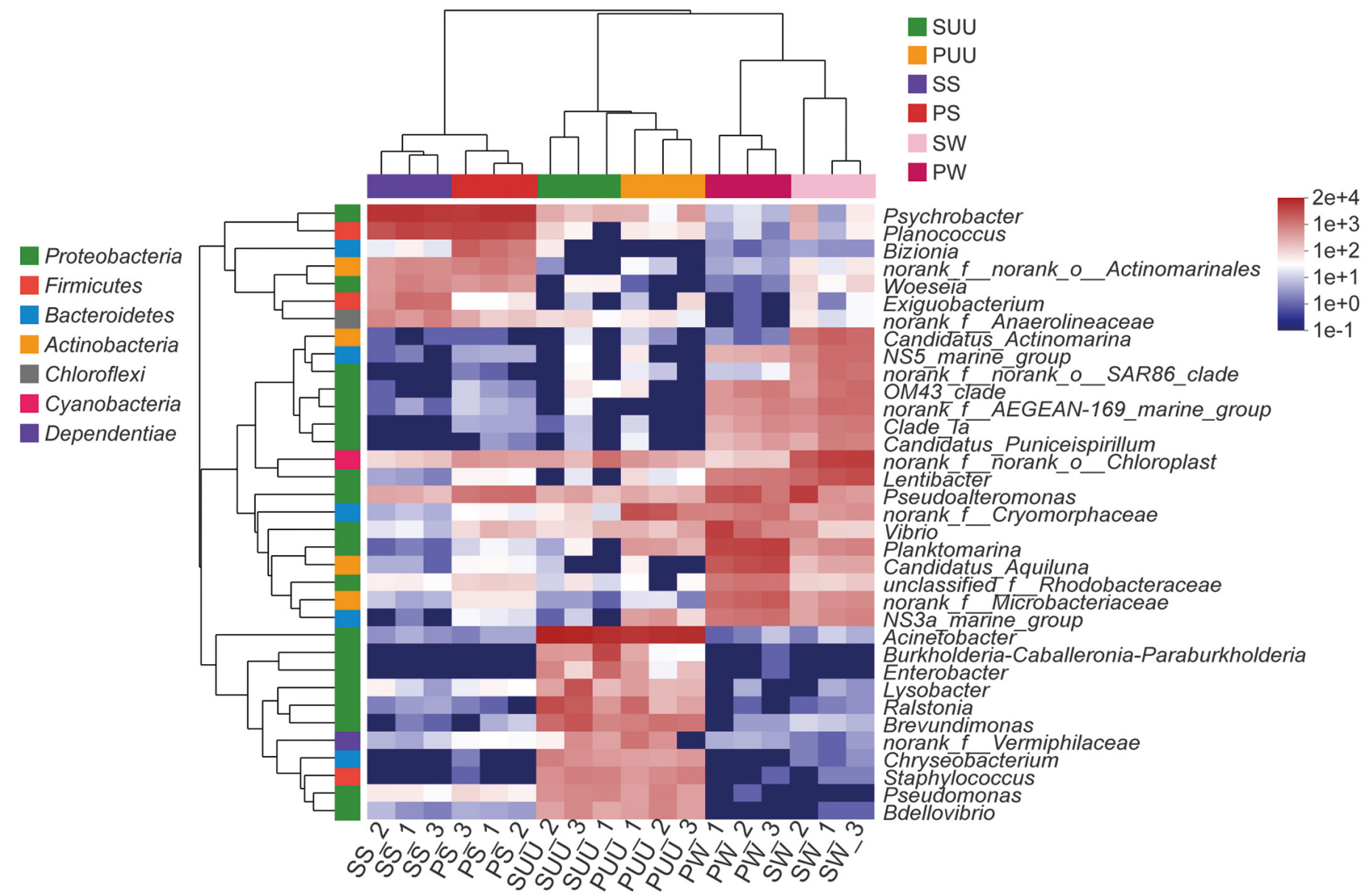

Fig. 2. Hierarchical clustering heat map at the genus level in samples collected from the intestine and habitats of Urechis unicinctus. The top row lists the sample group information (abbreviations as in Table 1). The left vertical column lists the cluster information, and the right vertical column lists the genera annotated by Silva. The heatmap plot depicts the relative abundance of each genus (vertical-axis clustering) within each group (horizontal-axis clustering). The $Z$-values, based on the abundance of gene sequences after normalization, are indicated by color intensity (legend indicated on the right side)

highest in PUU. Firmicutes were not found in the water samples, but were abundant in the sediment samples, whereas Actinobacteria were much higher in the water samples than in the other samples. Cyanobacteria were found almost exclusively in the SW samples. Chloroflexi and Acidobacteria were present in sediment samples (Fig. 1b).

Bacterial community composition in the intestine and the habitat samples at the genus level is displayed in Fig. 2, with a total of 1295 genera identified in all samples. The genus with the highest relative abundance in SUU (26.78\%) and PUU (22.10\%) was Acinetobacter. norank_f_Cryomorphaceae ${ }^{\mathbf{1}}$ accounted

\footnotetext{
1 Note on 'norank' in the nomenclature: In the comparison database, some intermediate grades of taxonomic lineages have no scientific names and are marked by NORANK. That is, the level is not named.
}

for $6.70 \%$ of all genera in PUU, while this relative abundance declined sharply in SUU (0.14\%). Psychrobacter and Planococcus were the major genera in SS and PS, accounting for 41.48 and $42.30 \%$ of all genera. The relative abundance of genera in the water samples presented a significant difference from the other samples. norank_f_norank_o_Chloroplast (14.48\%), Lentibacter (9.24\%) Pseudoalteromonas (7.07\%), and Candidatus_Aquiluna (5.39\%) were the top 4 genera in SW. Smaller percentages of Planktomarina (17.04\%), Candidatus_Aquiluna (12.65\%), Vibrio (9.28\%), Pseudoalteromonas (8.66\%), norank_ f_Microbacteriaceae $(7.44 \%), \quad$ NS3a_marine_group $(4.74 \%)$, unclasified_f_Rhodobacteraceae $(4.42 \%)$, and norank_f_Cryomorphaceae $(4.10 \%)$ were found in PW.

Additionally, the phylogenetic relationships of these genera differed (Fig. 2). Most genera belonged to Proteobacteria, including Psychrobacter, Candidatus_ 


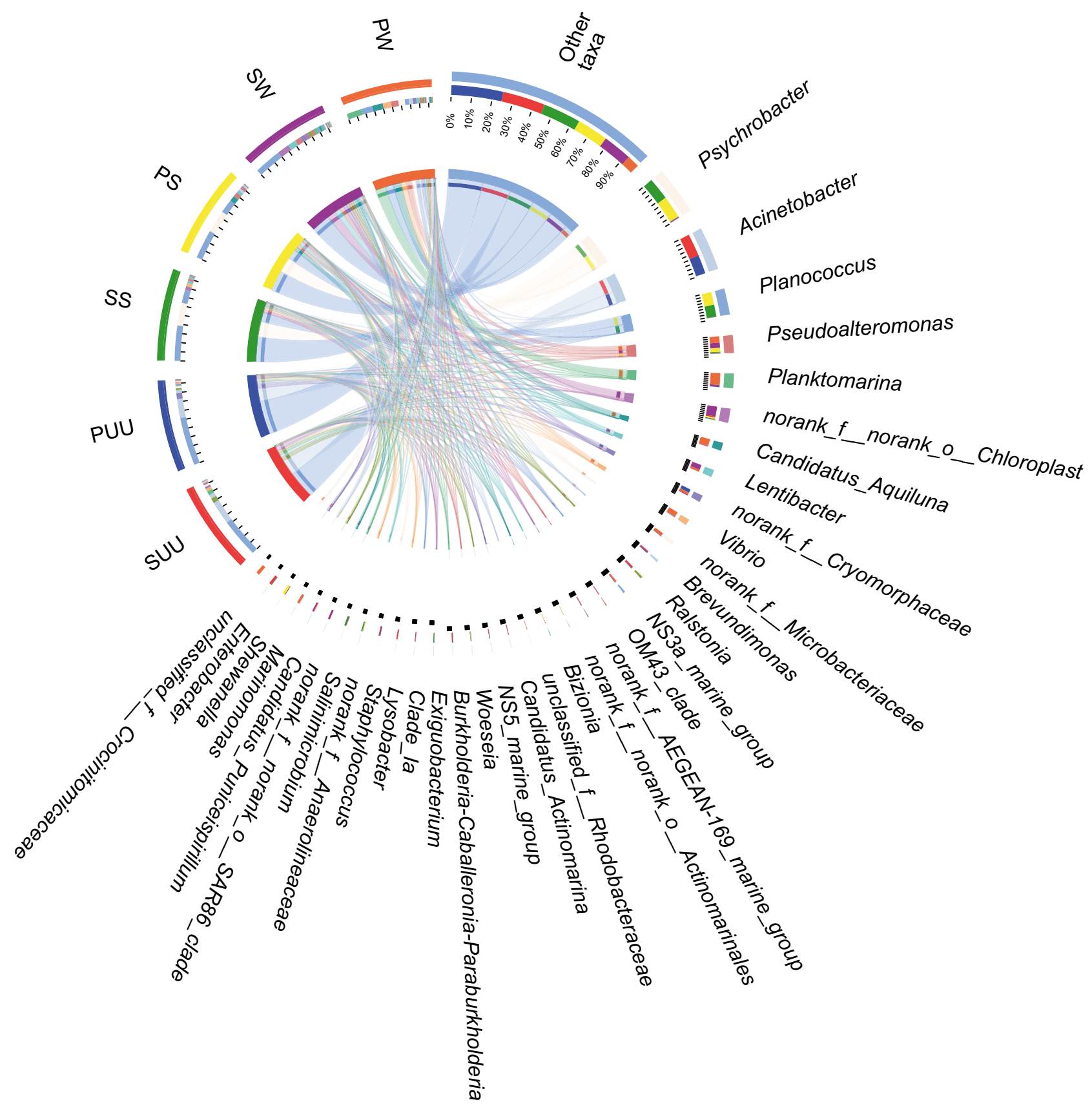

Fig. 3. Circos diagram of the relationship between samples and genera. The upper-left part of the circle shows the composition of genera in the samples (abbreviations as in Table 1); the colours of the outer band represent the samples, the colours of the inner band represent the genera, with the length of the colour sections indicating their relative abundances. The remaining part of the circle shows the samples in which genera occur; the outer band represents genera (each is given its own colour), the inner band represents different samples, with the length of the colour sections indicating their relative occurrences. The black tick marks provide a scale of proportion in $10 \%$ intervals. The central circle shows connections between genera and the samples in which they occur

Puniceispirillum, Pseudoalteromonas, Lentibacter, Acinetobacter, and Pseudomonas. Actinobacteria includes norank_f_norank_o_Actinomarinales, Candidatus_Actinomarina, Candidatus_Aquiluna and norank f_Microbacteriaceae. Planococcus, Exiguobacterium and Staphylococcus belong to Firmicutes. norank
f_Cryomorphaceae and NS3a_marine_group are members of Bacteroidetes.

The relationship between samples and species on the genus level is presented in a Circos diagram (Fig. 3). Acinetobacter was mainly found in the intestinal samples, and Psychrobacter and Planococcus 
were key species in the sediment samples. The genera found in water samples were diverse. The results were consistent with those shown in Fig. 2.

\subsection{Bacterial community analysis and comparison}

To compare bacterial community composition of the gut microbiota of $U$. unicinctus to those of their environments, we used principal co-ordinates analysis (PCoA) of weighted UniFrac distances (Fig. 4a). The 18 samples were separated into 3 clusters according to the second principal component (PC1) axis, accounting for $41.94 \%$ of the total variance. The gut microbiota (SUU and PUU) belonged to one group and were significantly different from the sediment samples (SS and PS) and the water samples (SW and PW). These groups are also consistent with the sample collection areas. Based on the UPGMA clustering tree (Fig. 4b), the taxonomic composition of all samples revealed 3 different groups, which was consistent with the results of the PCoA analysis. The gut microbiota (SUU, PUU) were clustered into one set in Fig. 4b. ANOSIM of all samples at the genus level also reflected that the species in intestinal samples were quite different from those in water and sediment samples (Fig. S4).

To detect species that exhibited differences in abundance in different microbial communities, the 15 top genera were selected to assess the significance of the observed differences in the intestine of $U$. unicinctus and the 2 different habitats using 1-way ANOVA (Fig. 5a). The abundances of Psychrobacter and Planococcus were extremely high in the sediment samples, which were significantly different from the other 2 groups. The genus Acinetobacter was only found in the intestine of Urechis unicinctus. norank_f_Cryomorphaceae was also found in PUU. The genera in water samples were significantly different from the other 2 groups. Planktomarina, Candidatus_Aquiluna, norank_f_Cryomorphaceae, and



Fig. 4. Relationships among microbiota of all the samples (group abbreviations as in Table 1). (a) Principal coordinates analysis (PCoA) showing the separate distribution of gut microbiota composition between sediment groups and seawater groups. The PC1 and PC2 axes explain 41.94 and $30.83 \%$ of the variation, respectively. Differences in community composition between samples from different groups were examined using ANOSIM, with 999 replacements. (b) UPGMA clustering tree at the phylum level among different groups

Vibrio were abundant in PW samples. The relative abundance of norank_f_norank_o_Chloroplast and Lentibacter was much higher in the SW samples. Pseudoalteromonas showed no obvious difference between SW and PW. To explore the different species inhabiting the intestines of $U$. unicinctus from the 2 different habitats, we again selected the 15 top genera to assess significance using Student's $t$-test (Fig. 5b). Except norank_f_Cryomorphaceae, the 

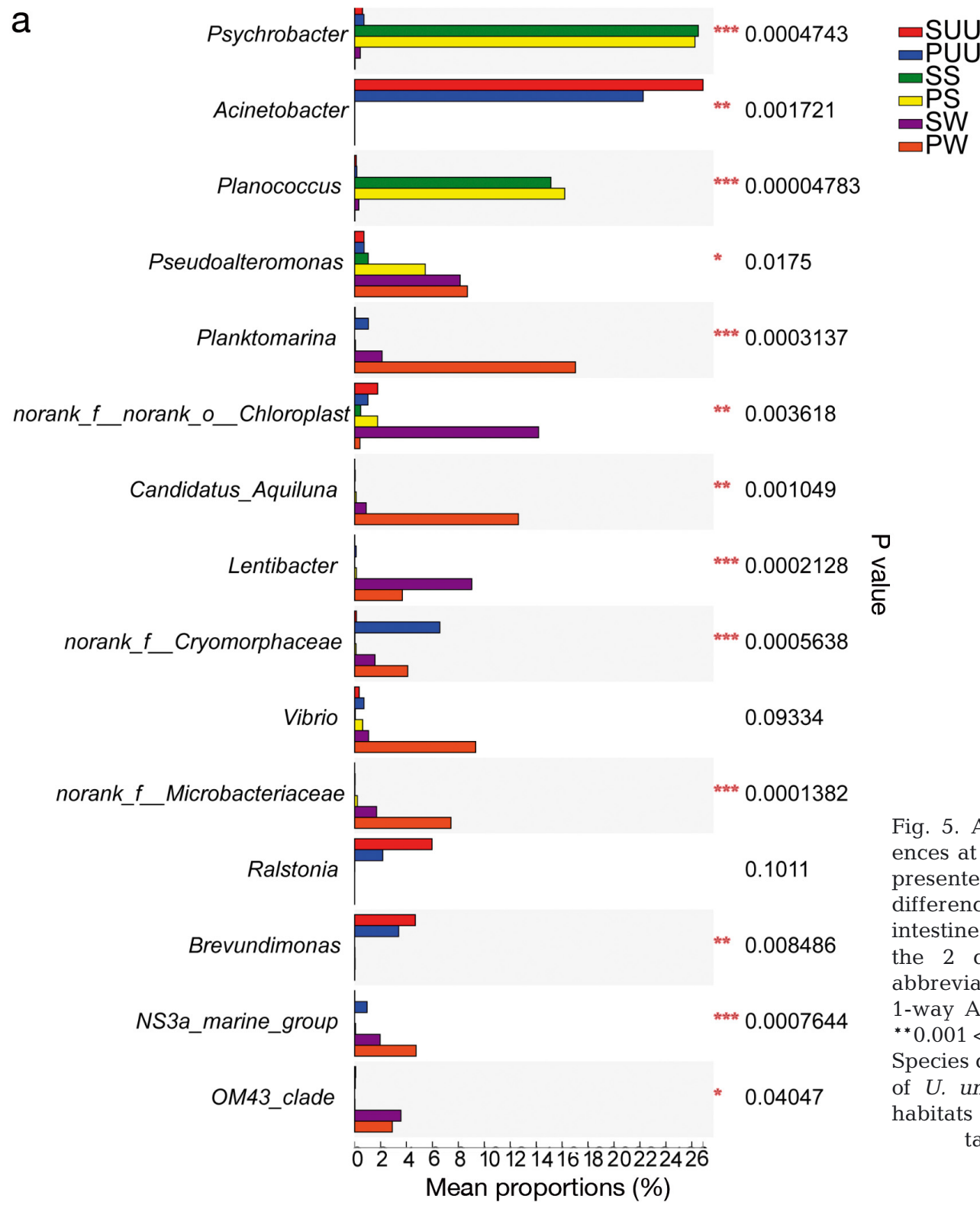

*** 0.00004783

* 0.0175

$* * * 0003137$

** 0.003618

** 0.001049

$0.0002128 \frac{\widehat{\varpi}}{\frac{0}{\Phi}}$

*** 0.0005638

0.09334

**** 0.0001382

0.1011

** 0.008486

Fig. 5. Analysis of species differences at the genus level. Data are presented as means. (a) Species differences in samples from the intestine of Urechis unicinctus and the 2 different habitats (group abbreviations as in Table 1) using 1 -way ANOVA $\left({ }^{*} 0.01<\mathrm{p} \leq 0.05\right.$; $\left.{ }^{* *} 0.001<\mathrm{p} \leq 0.01 ;{ }^{* * *} \mathrm{p} \leq 0.001\right)$. (b) Species differences in the intestine of $U$. unicinctus from 2 different habitats using Student's $t$-test (2tailed test ${ }^{*} \mathrm{p} \leq 0.05$ )

b

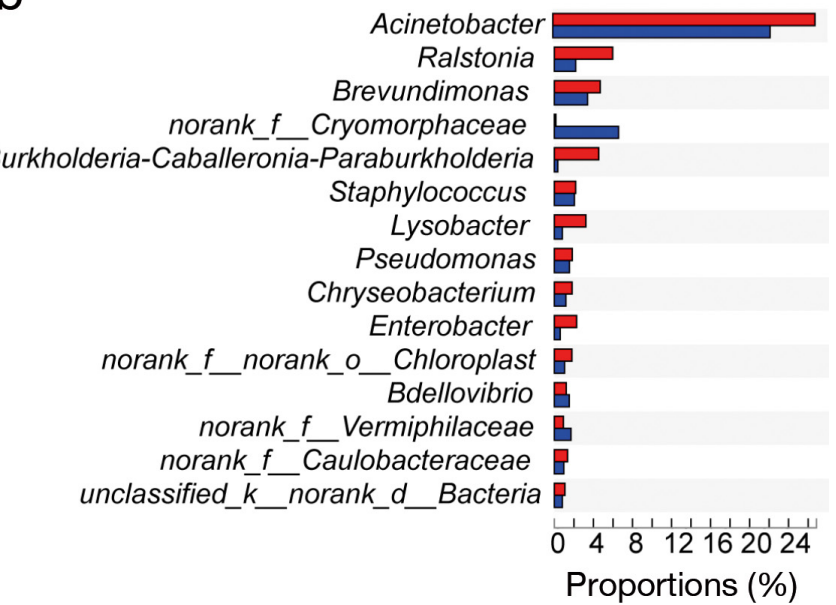

99\% confidence intervals

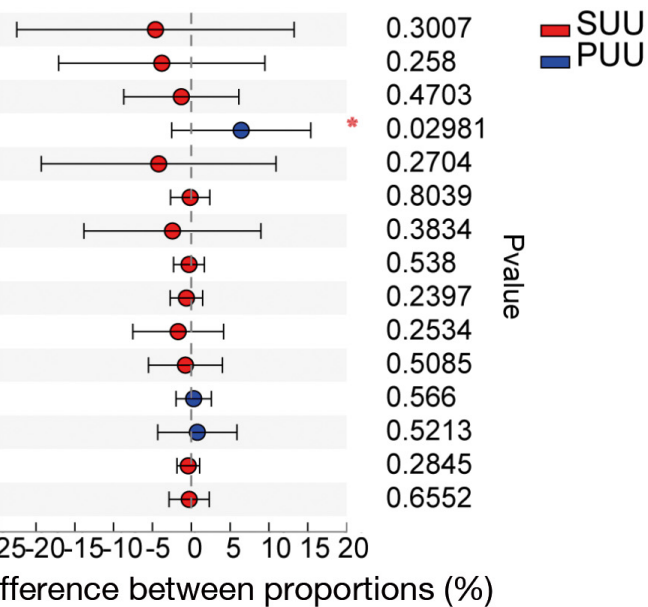


other 14 top genera showed no significant differences between the 2 intestinal samples. PUU and SUU had more species in common than the other samples did, indicating that the relationship between the 2 intestinal samples was much closer (Fig. S5). The results indicated that $U$. unicinctus can survive and grow well regardless of whether it is maintained in pond polyculture or in coastal mudflats.

\subsection{Functional analysis of intestinal microbial community}

COG analysis classified all intestinal microbial genes into 23 functional categories (Fig. 6) (Table S1). In this classification, except for Group S ('function unknown'), the largest group involved in the general function was amino acid transport and metabolism (Group E: $10.05 \%$ SUU, 10.08\% PUU), which was followed by translation, ribosomal structure, and biogenesis (Group J: $6.76 \%$ SUU, $7.32 \%$ PUU); energy production and conversion (Group C: $6.97 \%$ SUU, $6.88 \%$ PUU); cell wall/membrane/envelope biogenesis (Group M: $6.37 \%$ SUU, $6.73 \%$ PUU); inorganic ion transport and metabolism (Group P: $6.81 \%$ SUU, 6.46\% PUU); transcription (Group K: $5.99 \%$ SUU, $5.80 \%$ PUU); and carbohydrate trans- port and metabolism (Group G: 5.02\% SUU, 5.15\% PUU). Details are provided in Table S1.

The functional categories (KEGG level 2) of bacterial communities as predicted by PICRUSt analysis through a heatmap are shown in Fig. 7 \& Table S2. The metabolic pathways were the largest pathways (68.56\% PUU, 67.93\% SUU). Following metabolism, the important functions of gut microbiota were genetic information processing $(9.64 \%$ PUU, $8.85 \%$ SUU) and environmental information processing (8.36\% PUU, $9.31 \%$ SUU). The highest relative abundance of genes were related to carbohydrate metabolism (Table S3), including pyruvate metabolism, glyoxylate and dicarboxylate metabolism, amino sugar and nucleotide sugar metabolism, and fructose and mannose metabolism (Table S4, Fig. S6). In addition to carbohydrate metabolism, the genes for amino acid metabolism accounted for $11.7 \%$ of the total genes, including the genes to metabolize arginine, proline, glycine, serine, threonine, phenylalanine, tyrosine, alanine, aspartate, glutamate, cysteine, methionine and histidine. Interestingly, except for nitrogen metabolism and oxidative phosphorylation, methane metabolism was one of the main functions of energy metabolism (Fig. S7). The gut intestinal microbiota could also metabolize nucleotides (purine and pyrimidine), cofactors and

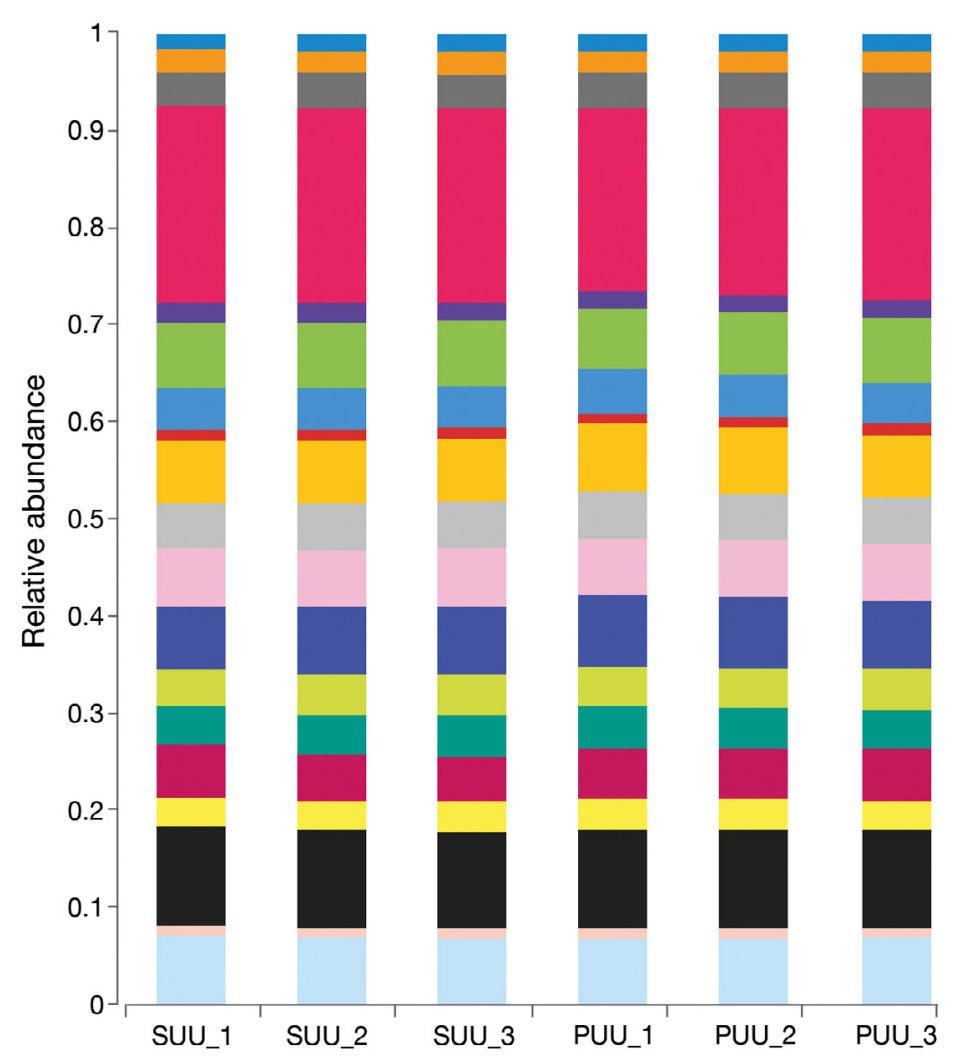

A : RNA processing and modification

$B$ : Chromatin structure and dynamics

$C$ : Energy production and conversion

$\mathrm{D}$ : Cell cycle control, cell division, chromosome partitioning

$\mathrm{E}$ : Amino acid transport and metabolism

$\mathrm{F}$ : Nucleotide transport and metabolism

$\mathrm{G}$ : Carbohydrate transport and metabolism

$\mathrm{H}$ : Coenzyme transport and metabolism

I : Lipid transport and metabolism

$\mathrm{J}$ : Translation, ribosomal structure and biogenesis

$\mathrm{K}$ : Transcription

$L$ : Replication, recombination and repair

$\mathrm{M}$ : Cell wall/membrane/envelope biogenesis

$\mathrm{N}$ : Cell motility

0 : Posttranslational modification, protein turnover, chaperones

$P$ : Inorganic ion transport and metabolism

$Q$ : Secondary metabolites biosynthesis, transport and catabolism

$S$ : Function unknown

$\mathrm{T}$ : Signal transduction mechanisms

$U$ : Intracellular trafficking, secretion, and vesicular transport

$V$ : Defense mechanisms

W : Extracellular structures

$\mathrm{Z}:$ Cytoskeleton
Fig. 6. Clusters of orthologous group (COG) function classification of the gut microbiota of Urechis unicinctus by PICRUSt analysis. Group abbreviations as in Table 1 


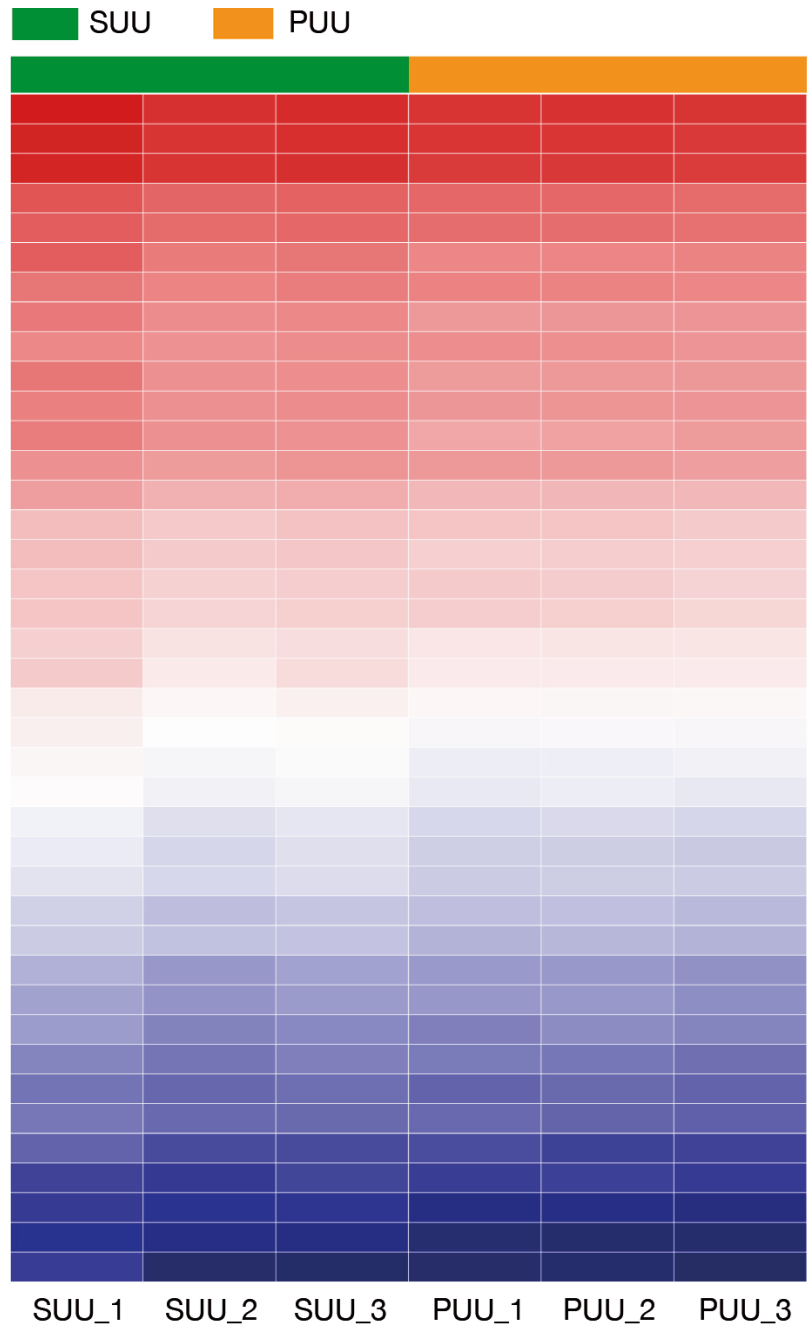

Carbohydrate metabolism

Global and overview maps

Amino acid metabolism

Energy metabolism

Metabolism of cofactors and vitamins

Membrane transport

Nucleotide metabolism

Signal transduction

Translation

Cellular community - prokaryotes

Lipid metabolism

Xenobiotics biodegradation and metabolism

Replication and repair

Metabolism of other amino acids

Folding, sorting and degradation

Metabolism of terpenoids and polyketides

Glycan biosynthesis and metabolism

Biosynthesis of other secondary metabolites

Drug resistance: Antimicrobial

Cell motility

Cell growth and death

Endocrine system

Infectious diseases: Bacterial

Cancers: Overview

Aging

Neurodegenerative diseases

Drug resistance: Antineoplastic

Transport and catabolism

Cardiovascular diseases

Endocrine and metabolic diseases

Immune system

Nervous system

Transcription

Environmental adaptation

Cancers: Specific types

Infectious diseases: Viral

Infectious diseases: Parasitic

Immune diseases

Excretory system

Digestive system

\section{SUU_1 SUU_2 SUU_3 PUU_1 PUU_2 PUU_3}

Fig. 7. Heatmap profiles showing the functional categories (KEGG level 2) of bacterial communities in the gut microbiota of Urechis unicinctus as predicted by PICRUSt analysis. Rows represent the 40 KEGG orthology (KO) functions, columns represent the 6 samples (SUU: coastal, PUU: pond polycultured), and the color intensity in the heatmap represents the relative abundance (log) of functional genes

vitamins, lipid, xenobiotics, terpenoids, polyketides, and glycan. Translation, replication, and repair and folding, sorting, and degradation were the main functions related to genetic information processing, with a relative abundance of approximately $9.2 \%$. The bacterial secretion system was of great significance for membrane transport, which was helpful for the gut microbiota to access to environmental information together with the signal transduction system, such as the $A B C$ transporter. Quorum sensing is a critical function for cellular communication in prokaryotes. Bacterial chemotaxis plays an important role in cell motility. The 2 functions above associated with cell growth and death were the top 3 functions of cellular processes $(6.4 \%)$ at KEGG level 2 . The genes for cationic antimicrobial peptide (CAMP) and vancomycin resistance were also found to relate to antimicrobial drug resistance. Platinum drug resistance and antifolate, which represent antineoplastic drug resistance, were among the functions of gut microbiota in $U$. unicinctus.

\section{DISCUSSION}

In this work, we first investigated the intestinal microbial community of Urechis unicinctus living in 2 different habitats. Compared to the studies of sea cucumbers (Zheng et al. 2018), the number of total effective OTUs in this study was much larger. We 
selected 18 samples, including 6 gut microbiota, to amplify the prokaryotic 16S rRNA gene, and 902871 pyrosequencing reads in total were generated. We identified 445-1783 prokaryotic OTUs at a 93\% sequence similarity level (Table 1).

Proteobacteria have a competitive edge in surviving in various ecological niches due to their variable morphology and versatile physiology. The relative abundance of Proteobacteria in the oceans was $57.9 \%$ (Shin et al. 2015), so they are a major phylum in intestinal samples of many marine organisms, including sea cucumbers (Zhang et al. 2018). Proteobacteria was the dominant phylum in the intestine of the sea cucumber Holothuria glaberrima ( $50 \%)$ (Weigel 2020). In our research, Proteobacteria were dominant in all samples, including intestinal samples of $U$. unicinctus, seawater, and sediment from 2 habitats. Bacteroidetes was the phylum with the second highest relative abundance in all samples. Bacteroidetes are able to produce extracellular enzymes with degradative capabilities (Huang et al. 2020), and they might contribute to carbohydrate metabolism (Weigel 2020). In this study, the abundance of Bacteroidetes was stable in the intestinal samples (SUU and PUU), indicating that the intestinal bacteria have adapted to the environmental stresses and help the host to decompose and metabolize the diet (Duan et al. 2020). However, the relative abundances of Firmicutes were almost undetectable in 2 water groups. The abundance of Firmicutes was higher in sediments than in the gut (Fig. 1b). Firmicutes, which include a number of beneficial bacteria, could be more effective for extracting energy from the diet, and they can prevent the production of inflammatory cytokines and pathogen-induced intestinal function disruption in fish (Duan et al. 2020). Bacteroidetes participate in the degradation of different biological polymers, such as cellulose, chitin, and pectin (Williams et al. 2013). Previous studies have demonstrated that an elevated ratio of Firmicutes to Bacteroidetes is associated with weight gain and metabolic disorders (Zhao et al. 2019). Therefore, the stable Firmicutes/Bacteroidetes ratio of intestinal samples (SUU and PUU) indicates that energy metabolism of $U$. unicinctus might be healthy. Due to their ability to produce various natural drugs, enzymes, and bioactive metabolites (Jiang 2013), Actinobacteria are promising potential probiotics in aquaculture (Das et al. 2008). Cyanobacteria presented a high relative abundance only in the SW samples.

At the genus level, several dominant genera exhibited large differences among groups. Psychrobacter had the highest relative abundance in the SS sam- ples. Psychrobacter is one of the dominant taxa in low-temperature environments that has the ability to degrade PAHs, is heavy-metal-resistant, and is presumably capable of denitrification in psychrophilic environments (Lasek et al. 2017). Acinetobacter represented over $20 \%$ of the relative abundance of the gut microbiota of SUU and PUU. Acinetobacter exhibit antibacterial activity against some tested human and fish pathogenic bacteria (Jami et al. 2015), and they are also resistant to many drugs (Wagenvoort et al. 2002), with defense mechanisms including efflux pumps, outer membrane porins, lipopolysaccharides, degradation enzymes ( $\beta$-lactamases, metalloproteinases, penicillin-binding proteins), and biofilm formation (Bonomo \& Szabo 2006, Livermore \& Woodford 2006). Few Acinetobacter can cause infectious diseases (de Breij et al. 2010), although researchers have verified that this genus might infect marine fishes and increase the threat of transmission from fishes to humans (Anand \& Suthindhiran 2020). The relative abundance of norank_f_Cryomorphaceae in PUU was significantly higher than that in SUU. Although little is known about this genus, its capacity to degrade proteins and algal storage polysaccharides has been confirmed (Grieb et al. 2020).

The physiological functions of hosts are greatly influenced by their intestinal microbiota (Goldsmith \& Sartor 2014). Except the 'function unknown' group, the largest group involved in the general function was amino acid transport and metabolism, followed by translation, ribosomal structure and biogenesis, energy production and conversion, cell wall/membrane/ envelope biogenesis, inorganic ion transport and metabolism, transcription and carbohydrate transport and metabolism based on the COG analysis. This is consistent with the literature (DeWitt \& Kudsk 1999). Based on the KEGG analysis, the largest group was amino acid and carbohydrate metabolism, indicating that intestinal microbes might prefer a diet rich in carbohydrates and proteins. Metabolism was the main function of the gut microbiota in $U$. unicinctus. In addition to nutrient metabolism, they also had capacity to degrade some harmful substances in the host including xenobiotics such as terpenoids and polyketides. Gut microorganisms can provide energy from digestion and metabolism based on multiple energy metabolism pathways (Webster 1980), such as nitrogen, oxidative phosphorylation, and methane metabolism.

Membrane transport and signal transduction are important for bacteria to access environmental information and conduct biological processes (CornejoGranados et al. 2017). The microbial genes involved 
in membrane transport and signal transduction were abundant in the gut samples from $U$. unicinctus. These functional genes could also be found in tilapia (Wu et al. 2020). The genes encoding CAMP, vancomycin, platinum drug resistance, and antifolate resistance were also found among gut microbiota to tolerate the pathogenic bacteria and drugs, which indicated that the gut microbiota could modulate and enhance the immune system of $U$. unicinctus along with Th17 cell differentiation and the IL-17 signaling pathway (Belkaid \& Hand 2014). Some other important pathways, including glucagon, insulin, estrogen, and adipocytokine signaling pathways and thyroid hormone synthesis and signaling pathways, suggested a vital role of the intestinal microbiota in the modulation of the endocrine system. The genes encoding GABAergic synapse (ko04727) and glutamatergic synapse (ko04724) were included in the KEGG analysis of gut microbes on level 3 (Table S4). Bacterial chemotaxis, flagellar assembly, quorum sensing, and biofilm formation could support the gut microorganism moving and communicating among the cellular processes to adapt to the intestinal environment of the host. These results may be of reference value to the poly-aquaculture management of $U$. unicinctus with other aquaculture organisms.

\section{CONCLUSION}

This study represents the first high-throughput absolute abundance quantification analysis of the gut microbiota diversity and functions in Urechis unicinctus from 2 different habitats. We found that Proteobacteria, Bacterioidetes, Firmicutes, and Actinobacteria were the dominant phyla in the gut microbial community, and the top genera were Acinetobacter, Ralstonia, and Brevundimonas. Psychrobacter and Planococcus were significantly high in sediment samples, while in sea water samples, Planktomarina, norank_f_norank_o_Chloroplast, and Candidatus_Aquiluna were the dominant genera. The gut microbiota were significantly different from the SS and SW samples. The major composition of gut bacteria did not show significant differences among the top 15 genera except norank_f_Cryomorphaceae in PW and SW.

The functions of intestinal microbial community largely centered on metabolism. In addition to the main carbohydrate and amino acid metabolism, energy metabolism including nitrogen metabolism, oxidative phosphorylation, and methane metabolism were relatively important. The gut microbiota could access information from the intestinal environment by virtue of membrane transport and signal transduction systems. The findings in the present study provide an understanding of the gut microbiome composition and diversity in $U$. unicinctus from 2 different habitats. We found no significant differences in the gut microbiota, indicating that U. unicinctus can survive and grow both in pond polyculture as well as in coastal mudflats, which can be instructive for the management of animal health in $U$. unicinctus farming activities, and for further gut microecology research.

Acknowledgements. This research was financially supported by the Shandong Provincial Natural Science Foundation (Grant number: ZR2018MD018), Yantai University Doctoral Start-up Foundation (Grant number: HX2018B32), and Shandong Key Laboratory of Marine Ecological Restoration (Grant number: 201919).

\section{LITERATURE CITED}

Anand KP, Suthindhiran K (2020) Biofilm formation and pathogenicity of marine-derived Acinetobacter sp. VITRSA1 in Paratelphusa hydrodromous and its toluene remediation. Biocatal Agric Biotechnol 28:101723

Belkaid Y, Hand TW (2014) Role of the microbiota in immunity and inflammation. Cell 157:121-141

* Bi Q, Chu J, Feng Y, Jiang Z, Han B, Liu W (2013) Purification and characterization of a new serine protease with fibrinolytic activity from the marine invertebrate, Urechis unicinctus. Appl Biochem Biotechnol 170:525-540

Bonomo RA, Szabo D (2006) Mechanisms of multidrug resistance in Acinetobacter species and Pseudomonas aeruginosa. Clin Infect Dis 43(Suppl 2):S49-S56

Chen W, Zhang S, Xu Y, Sun Y, Song L, Tian B, Liu T (2020) Effects of stocking density on the growth performance, physiological response and intestinal microbiota of juvenile Echiura worms (Urechis unicinctus). Aquacult Res 51:3983-3992

Cornejo-Granados F, Lopez-Zavala AA, Gallardo-Becerra L, Mendoza-Vargas A and others (2017) Microbiome of Pacific whiteleg shrimp reveals differential bacterial community composition between wild, aquacultured and AHPND/EMS outbreak conditions. Sci Rep 7:11783

Das S, Ward L, Burke C (2008) Prospects of using marine actinobacteria as probiotics in aquaculture. Appl Microbiol Biotechnol 81:419-429

* de Breij A, Dijkshoorn L, Lagendijk E, van der Meer J and others (2010) Do biofilm formation and interactions with human cells explain the clinical success of Acinetobacter baumannii? PLOS ONE 5:e10732

* DeWitt RC, Kudsk KA (1999) The gut's role in metabolism, mucosal barrier function, and gut immunology. Infect Dis Clin North Am 13:465-481

Duan Y, Xiong D, Li Y, Dong H, Wang W, Zhang J (2020) Changes in the gastrointestinal microbial community of Lateolabrax maculatus in a naturally occurring Microcystis aeruginosa bloom environment. Aquaculture 528: 735444 
Goldsmith JR, Sartor RB (2014) The role of diet on intestinal microbiota metabolism: downstream impacts on host immune function and health, and therapeutic implications. J Gastroenterol 49:785-798

* Grieb A, Francis TB, Krüger K, Orellana LH, Amann R, Bernhard MF (2020) Candidatus Abditibacter, a novel genus within the Cryomorphaceae, thriving in the North Sea. Syst Appl Microbiol 43:126088

*Huang L, Guo H, Chen C, Huang X and others (2020) The bacteria from large-sized bioflocs are more associated with the shrimp gut microbiota in culture system. Aquaculture 523:735159

Huttenhower C, Gevers D, Knight R, Abubucker S and others (2012) Structure, function and diversity of the healthy human microbiome. Nature 486:207-214

Jami M, Ghanbari M, Kneifel W, Domig KJ (2015) Phylogenetic diversity and biological activity of culturable Actinobacteria isolated from freshwater fish gut microbiota. Microbiol Res 175:6-15

Jiang XT, Peng X, Deng GH, Sheng HF, Wang Y, Zhou HW, Tam NFY (2013) Illumina sequencing of 16S rRNA tag revealed spatial variations of bacterial communities in a mangrove wetland. Microb Ecol 66:96-104

Jumars PA, Dorgan KM, Lindsay SM (2015) Diet of worms emended: an update of polychaete feeding guilds. Annu Rev Mar Sci 7:497-520

Kong Y, Li M, Chu G, Liu H, Shan X, Wang G, Han G (2021) The positive effects of single or conjoint administration of lactic acid bacteria on Channa argus: digestive enzyme activity, antioxidant capacity, intestinal microbiota and morphology. Aquaculture 531:735852

Langille MGI, Zaneveld J, Caporaso, JG, McDonald D and others (2013) Predictive functional profiling of microbial communities using 16S rRNA marker gene sequences. Nat Biotechnol 31:814-821

Kasek R, Dziewit L, Ciok A, Decewicz P and others (2017) Genome content, metabolic pathways and biotechnological potential of the psychrophilic Arctic bacterium Psychrobacter sp. DAB_AL43B, a source and a host of novel Psychrobacter-specific vectors. J Biotechnol 263:64-74

K Ley RE, Bäckhed F, Turnbaugh P, Lozupone CA, Knight RD, Gordon JI (2005) Obesity alters gut microbial ecology. Proc Natl Acad Sci USA 102:11070-11075

K Liu B, Zhou W, Wang H, Li C, Wang L, Li Y, Wang J (2020) Bacillus baekryungensis MS1 regulates the growth, nonspecific immune parameters and gut microbiota of the sea cucumber Apostichopus japonicus. Fish Shellfish Immunol 102:133-139

Livermore DM, Woodford N (2006) The beta-lactamase threat in Enterobacteriaceae, Pseudomonas and Acinetobacter. Trends Microbiol 14:413-420

Lu J, Zhang Y, Wu J, Wang J (2020) Nitrogen removal in recirculating aquaculture water with high dissolved oxygen condition using the simultaneous partial nitrification, anammox and denitrification system. Bioresour Technol 305:123037

Ma X, Liu X, Zhou D, Bai Y, Gao B, Zhang Z, Qin Z (2016) The NF-kB pathway participates in the response to sulfide stress in Urechis unicinctus. Fish Shellfish Immunol 58:229-238

Mouchet MA, Bouvier C, Bouvier T, Troussellier M, Escalas A, Mouillot D (2012) Genetic difference but functional similarity among fish gut bacterial communities through molecular and biochemical fingerprints. FEMS Microbiol Ecol 79:568-580
Parris DJ, Morgan MM, Stewart FJ (2019) Feeding rapidly alters microbiome composition and gene transcription in the clownfish gut. Appl Environ Microbiol 85: e02479-18

* Patil Y, Gooneratne R, Ju X (2020) Interactions between host and gut microbiota in domestic pigs: a review. Gut Microbes 11:310-334

* Shi X, Shao M, Zhang L, Ma Y, Zhang Z (2012) Screening of genes related to sulfide metabolism in Urechis unicinctus (Echiura, Urechidae) using suppression subtractive hybridization and cDNA microarray analysis. Comp Biochem Physiol Part D Genomics Proteomics 7:254-259

Shin NR, Whon TW, Bae JW (2015) Proteobacteria: microbial signature of dysbiosis in gut microbiota. Trends Biotechnol 33:496-503

Song X, Feng Z, Zhang Y, Zhu W (2019) Regulation of dietary astragalus polysaccharide (APS) supplementation on the non-specific immune response and intestinal microbiota of sea cucumber Apostichopus japonicus. Fish Shellfish Immunol 94:517-524

*Tarnecki AM, Burgos FA, Ray CL, Arias CR (2017) Fish intestinal microbiome: diversity and symbiosis unraveled by metagenomics. J Appl Microbiol 123:2-17

* Tremaroli V, Bäckhed F (2012) Functional interactions between the gut microbiota and host metabolism. Nature 489:242-249

*Wagenvoort J, De Brauwer E, Toenbreker H, van der Linden C (2002) Epidemic Acinetobacter baumannii strain with MRSA-like behaviour carried by healthcare staff. Eur J Clin Microbiol Infect Dis 21:326-327

*Wang M, Monaco MH, Donovan SM (2016) Impact of early gut microbiota on immune and metabolic development and function. Semin Fetal Neonatal Med 21:380-387

*Wang Y, Shi T, Huang G, Gong J (2018) Molecular detection of eukaryotic diets and gut microbiomes in two marine sediment-dwelling worms, Sipunculus nudus and Urechis unicinctus. Microbes Environ 33:290-300

WWebster AJF (1980) Energy costs of digestion and metabolism in the gut. In: Ruckebusch Y, Thivend P (eds) Digestive physiology and metabolism in ruminants. Springer, Dordrecht, p 469-484

*Weigel BL (2020) Sea cucumber intestinal regeneration reveals deterministic assembly of the gut microbiome. Appl Environ Microbiol 86:e00489-20

*Wilkes Walburn J, Wemheuer B, Thomas T, Copeland E and others (2019) Diet and diet-associated bacteria shape early microbiome development in yellowtail kingfish (Seriola lalandi). Microb Biotechnol 12:275-288

*Williams TJ, Wilkins D, Long E, Evans F, DeMaere MZ, Raftery MJ, Cavicchioli R (2013) The role of planktonic Flavobacteria in processing algal organic matter in coastal East Antarctica revealed using metagenomics and metaproteomics. Environ Microbiol 15:1302-1317

*Winter SE, Bäumler AJ (2014) Dysbiosis in the inflamed intestine: Chance favors the prepared microbe. Gut Microbes 5:71-73

Wu Z, Wang S, Zhang Q, Hao J, Lin Y, Zhang J, Li A (2020) Assessing the intestinal bacterial community of farmed Nile tilapia (Oreochromis niloticus) by high-throughput absolute abundance quantification. Aquaculture 529: 735688

Xu J, Wang R, Li L, Wang J (2020) Effective inhibition of ammonium released from heavily contaminated sediments through selective oxidation with zeolite layer. Geomicrobiol J 37:287-300 
Yang G, Tian X, Dong S (2019) Bacillus cereus and rhubarb regulate the intestinal microbiota of sea cucumber (Apostichopus japonicus Selenka): species-species interaction, network, and stability. Aquaculture 512:734284

Yuan X, Zhou Y, Mao Y (2015) Apostichopus japonicus: a key species in integrated polyculture systems. Dev Aquacult Fish Sci 39:323-332

Zhang Z, Xing R, Lv Z, Shao Y, Zhang W, Zhao X, Li C (2018) Analysis of gut microbiota revealed Lactococcus garviaeae could be an indicative of skin ulceration syndrome in farmed sea cucumber Apostichopus japonicus. Fish Shellfish Immunol 80:148-154

Zhao Y, Liu H, Wang Q, Li B, Zhang H, Pi Y (2019) The effects of benzo[a]pyrene on the composition of gut microbiota and the gut health of the juvenile sea cucum-

Editorial responsibility: Vengatesen Thiyagarajan, Hong Kong $S A R$

Reviewed by: 3 anonymous referees ber Apostichopus japonicus Selenka. Fish Shellfish Immunol 93:369-379

K Zhao Z, Jiang J, Pan Y, Dong Y and others (2020) Temporal dynamics of bacterial communities in the water and sediments of sea cucumber (Apostichopus japonicus) culture ponds. Aquaculture 528:735498

* Zheng Y, Wu W, Hu G, Qiu L and others (2018) Gut microbiota analysis of juvenile genetically improved farmed tilapia (Oreochromis niloticus) by dietary supplementation of different resveratrol concentrations. Fish Shellfish Immunol 77:200-207

* Zheng X, Yang R, Hu J, Lin SQ, Gu ZF, Ma ZH (2019) The gut microbiota community and antioxidant enzymes activity of barramundi reared at seawater and freshwater. Fish Shellfish Immunol 89:127-131

Submitted: September 15, 2020

Accepted: January 19, 2021

Proofs received from author(s): May 12, 2021 